\title{
Pulmonary mucoepidermoid carcinoma: Clinicopathological characteristics of 20 cases and a literature review
}

\section{Jing Zhang}

East Hospital

\section{Yan Xiao Chen}

Shanghai Jiao Tong University Medical School Affiliated Ruijin Hospital

Jun Zi Qian

Shanghai Jiao Tong University Medical School Affiliated Ruijin Hospital

\section{Ping Yue}

Shanghai Jiao Tong University Medical School Affiliated Ruijin Hospital

Jialei Wang

Shanghai Jiao Tong University Medical School Affiliated Ruijin Hospital

Yi Xiang ( $\nabla$ xiangyiht@163.com )

https://orcid.org/0000-0002-0724-6359

\section{Research article}

Keywords: pulmonary mucoepidermoid carcinoma clinicopathology

Posted Date: February 5th, 2020

DOI: https://doi.org/10.21203/rs.2.17790/v2

License: (c) (1) This work is licensed under a Creative Commons Attribution 4.0 International License. Read Full License 


\section{Abstract}

Background: Pulmonary mucoepidermoid carcinoma is a rare tumor of the lung. The clinicopathological characteristics of pulmonary mucoepidermoid carcinoma are not well defined due to the low incidence. This study was performed to provide more supplementary clues for the identification and understanding of pulmonary mucoepidermoid carcinoma. Methods: We reviewed the medical records since January 1, 2000 to December 31, 2018. The patients' medical records $\llbracket$ including age at the time of diagnosis , gender, smoking history, preoperative evaluations, operative procedures, tumor location, tumor size, tumor stage, lymph node metastasis, pathological markers, prognosis and survival information were extracted and reviewed. Categorical variables were presented as parameters and percentages. A comparison was performed between patients with high and low grade of pulmonary mucoepidermoid carcinoma. Results: 20 patients were identified and the age span is from 18 to 67 year-old with the average age is 45 . Mucoepidermoid carcinomas were commonly found in men $₫ 60 \% \rrbracket .80 \%$ patients had clinical presentations and the positive rate of tumor markers was $78 \%$, although no specific tumor markers were found. TTF-1 were negative in all cases. ALK rearrangement was identified in a non-smoking woman with high grade pulmonary mucoepidermoid carcinoma. Surgery is the main procedure. 3-year survival rate is $72 \%$ and $80 \%$ patients achieved disease-free alive. High-grade patients tend to harbor older age $(p=0.035)$, larger tumor volume $(p=0.026)$ and higher index of $k i-67(p=0.0005)$. Conclusions: Pulmonary mucoepidermoid carcinoma could occur in a wide age span. Early diagnosis and complete surgical resection may promise a good prognosis. Grading is a key factor to predict the overall survival time. Combined TTF-1 and MAML2 will benefit the identification of pulmonary mucoepidermoid carcinoma from other lung tumors. Future prospective randomized controlled trials and larger, multi-centric series are needed.

\section{Background}

Pulmonary mucoepidermoid carcinoma (PMEC) is a rare tumor that accounts for $0.1 \%$ to $0.2 \%$ of all pulmonary tumors. Most pulmonary MECs arise in the proximal bronchi [1]. Histologically, the classification of the PMEC is mainly based on the degree of mitosis $\square$ necrosis and abnormalities of the nucleus in the tumor. Low-grade mucoepidermoid carcinoma consists mainly of glandular-like structures and mucin-secreting cells, whereas high-grade PMECs include a large number of squamous cells [2]. However, in a few previous studies, intermediated grade had been suggested because of different grade systems described by Brandwein[3]. So far, most researches on PMEC belonged to either a series of cases report or small sample retrospective studies, especially in China [4-6]. The characteristics of PMEC are not well defined due to the low incidence of PMEC and there are a lot of controversies in the diagnosis and treatment of PMEC. In this paper, we analyzed the clinicopathological features, treatment and prognostic information of 20 PMEC patients who were exposed to 2000-2018 in Shanghai Ruijin Hospital and Shanghai Cancer center. This study was performed to provide more information for the identification and understanding of pulmonary mucoepidermoid carcinoma. 


\section{Methods}

\section{Patients and tissue specimens}

The study was conducted under the approval by the Institutional Review Board. Ethical approval was granted by the clinical Research Ethics Committee of Shanghai Ruijin Hospital, Jiaotong University and Shanghai Cancer center, Fudan university. We reviewed the medical records, and outpatient pathology visits since January 1, 2000 to December 31, 2018 and collected 20 patients with PMEC, including 12 inpatients and 8 cases of pathology consultation patients whose data were partly missing. Signed informed consent was obtained from all induced patients for the acquisition and use of tissue samples and anonymized clinical data. Tissue specimens were collected from surgical resection or biopsy. The bronchoscopy was performed in 8 inpatients. Two inpatients had a diagnosis of PMEC by bronchoscopy, whereas the other patients were finally diagnosed through resected specimens. Hematoxylin and eosin(H\&E) stained slides in all patients were reviewed by two experienced pathologists for histopathological confirmation. PMEC was divided into low or high grade according to the World Health Organization(WHO) criteria of lung cancer [7].

\section{Data collection and extraction}

The patients' medical records囚including age at the time of diagnosis, gender, smoking history, preoperative evaluations, operative procedures, tumor location, tumor size, tumor stage, lymph node metastasis, pathological markers, prognosis and survival information were extracted and reviewed. Tumor stages were classified according to the American Joint Committee on Cancer Staging System, 7th edition. We acquired follow-up data from outpatient follow-ups or telephone and the last follow up time is December 31, 2018.

\section{Statistical analysis}

Categorical variables were presented as parameters and percentages. Nominal variables included gender, smoker, lymph node metastasis and TNM stage were conducted using Fisher's exact test. Age, tumor size and Ki-67 labeling index were compared by way of an independent-samples t-test. All tests were twosided, and p-values less than 0.05 were considered to be statistically significant.

\section{Results}

\section{Clinical characteristics}

Table. 1 summarized the main clinical features of the 20 patients. Twenty cases of PMEC included 8 females and 12 males ranged from 18 to 67 years old with a mean age of 45 . Five of 13 patients were smokers. Among ten patients, eight had symptoms which were presenting as cough(7/8) $\square$ fever(1/8) $\square$ hemoptysis(1/8) and bone pain(1/8). Two patients were asymptomatic. All 12 inpatients underwent CT examination. CT imaging showed a solitary nodule located at the periphery or the hilum of lung (Figure 
1). Almost half of patients (42\%) had implications, presented as atelectasis or obstructive pneumonia. 8 patients performed bronchoscopy and the neoplasm grew into the lumen in 7(88\%) patients. In our series, two cases with low grade PMEC had lymph node metastasis. One case with high grade occurred lymph node and bone metastasis.13(65\%) tumors were located in right lung, including right upper lobe( $\mathrm{n}=2)$, right middle lobe $(n=5)$, right lower lobe $(n=6) .7(35 \%)$ tumors were located in left lung, including left upper lobe $(n=4)$, left lower lobe $(n=3)$. The levels of serum tumor markers were detected in 9 patients. $7(78 \%)$

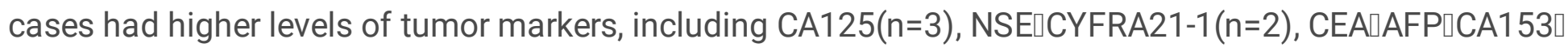
$\operatorname{SCCA}(n=1)$, while two(22\%) patients were normal.

\section{Histopathology}

Twenty patients were divided into low-grade (85\%) and high-grade (15\%) according to pathological diagnosis (Figure 2). Low-grade PMECs show cystic patterns with solid areas, three cell types: bland columnar mucin-secreting cells, squamoid cells, and intermediate cells, with rare necrosis. While, high grade PMECs mainly show solid areas, which composed of atypical squamoid cells, accompanied by variable numbers of mucin-secreting cells, with frequent mitosis and necrosis. Some patients' pathological characteristics were available and summarized in Table2. The results showed that resection margins were negative in all $14(100 \%)$ patients and no perineural invasion were found in $6(100 \%)$ cases who had been recorded. Intravascular embolus were found in 2(33\%) cases. Pleural involvement were positive in $4(36 \%)$ patients among 11 patients. Bronchus invasion existed in 2 (21\%)patients. TTF- 1 were negative in all patients and P63 were detected in $67 \%$ patients.

\section{Treatment}

The main treatment was surgery (Table1). Operation procedures included $12(63 \%)$ lobectomies, 4 (21\%)bilobectomies, $1(5 \%)$ sleeve resection and 2(11\%) pneumonectomies. However, there was one patient with high grade PMEC lost the opportunity of surgery because of bone metastasis.

Chemotherapies were performed in 3(25\%)patients after operation among 12 cases and the patient accompanied with bone metastasis received local radiotherapy. 11 patients had follow-up records and the follow-up time ranged from 8 to 126 months (mean 52m). Two patients died of tumor at 8 and 18 months after diagnosis. Among these two patients, one did not undergo surgery because of bone metastasis and the other one suffered recurrence in ipsilateral lung after surgery. The remaining 9 patients were alive without evidence of recurrence or metastasis. A comparison was performed between patients with high and low grade of pulmonary mucoepidermoid carcinoma. There were statistically significant differences in the age $(p=0.035)$, tumor size $(p=0.026)$ and the index of ki-67 $(p=0.0005)$.

\section{Discussion}

Based on the WHO Classification of lung tumors (2015), primary salivary gland-type tumors(pSGTs) can be divided into the following four types: mucoepidermoid carcinoma (MEC), adenoid cystic carcinoma (ACC), epithelial-myoepithelial carcinoma (EMC), and pleomorphic adenocarcinoma (PA) [8]. Pulmonary mucoepidermoid carcinoma is the most common type of pSGTs, followed by PACC [9]. In clinic, the age 
range of PACC had been reported to be 21-80. PMEC had a broad spectrum in age from 3 to 78 years old and often occurred in 40s $[5,10]$. According to the study conducted by Kumar, PACC was mostly localized to the trachea and main bronchus, while PMEC was more commonly diagnosed in the lung. Patients with PMEC had a better prognosis than PACC. The age span of our series is from 18 to 67 year-old and the average age is 45 . Interestingly, in accordance with recent reports, we also found that PMECs were commonly found in men [11]. 80\% patients had clinical presentations and the positive rate of tumor markers was $78 \%$, although no specific markers were found. Chest CT had an important value in the diagnosis of PMEC despite rare tumors exhibited an endobronchial pattern. If these patients were accompanied with persistent respiratory symptoms or elevated tumor markers, bronchoscopy examination and biopsy should be conducted to avoid misdiagnosis. TTF-1 was negative in all PMECs, conforming that it was of great value in distinguishing PMEC from lung adenocarcinoma, adenosquamous carcinoma and PMEC-like tumors [12]. Surgery is the main procedure of PMEC. Most patients were alive without evidence of disease after completely resection [13]. Our study showed that 3year survival rate of PMEC is $72 \%$, and 80 percent of those patients achieved disease-free alive. Histological grade was widely recognized as a prognostic factor. Our series also demonstrated that highgrade PMEC patients tend to harbor older age, larger tumor volume and higher index of ki-67. Due to the small size of samples, we did not find significant difference in TNM stage and metastasis between low and high grade of PMEC. However, some large series had revealed the worse outcomes of patients who accompanied with advanced TNM staging and earlier metastasis [14-16].

Accumulated evidences demonstrated that earlier diagnosis and complete surgical resection would benefit prognosis of PMEC. Histological examination is the most reliable way to distinguish it from other PMEC-like tumors and confirm its differentiation grade. It is worth mentioning that in small biopsies the distinction between PMEC and mucous gland adenoma can be very difficult. Mucous gland adenoma is a benign tumor composed of acini lined by mucus-containing cuboidal cells, while PMEC is a malignant tumor contain three cell types $₫$ mucin-secreting, squamoid, and intermediate cells. The absence of squamous and intermediate cell, immunohistochemically negativity for cytokeratin 5/6खP63 and P40, lack of malignancy features such as cellular atypia, mitotic activity and invasive growth pattern suggests mucous gland adenoma. Poorly differentiated mucoepidermoid carcinoma is sometimes difficult to distinguish from squamous carcinoma in the form of HE under the microscope. The differential diagnosis of high grade PMEC from squamous carcinoma may depend on: (1) surface epithelium lacking the changes of carcinoma in situ; (2) absence of individual cell keratinization and squamous pearl formation; (3) the presence of transitional areas to low-grade mucoepidermoid carcinoma or scattered mucin-secreting cells.

At present, there were few studies focused on driven genes of PMEC. Evidences indicated that the most frequent gene mutation in PMEC is MAML2, which belongs to MasterMind-like gene family on chromosome 11q21. In salivary gland tumors, MAML2 rearrangement has been identified in $40-75 \%$ of mucoepidermoid carcinoma [17-18]. However, the association between MAML2 rearrangement and prognosis is still in suspense. The current view suggests that MALML2 is more prone to occur in lowergrade PMEC and has no value for prognosis [19-21]. PMEC has a low preoperative diagnosis rate through 
bronchoscopy and CT-guide percutaneous lung puncture biopsy. Most of our cases had been treated as non small cell lung cancer (NSCLC) before and during surgery. It suggested that the application of molecular techniques to detect the MAML2 rearrangement and TTF-1 expression may be helpful in improving the preoperative diagnosis. Targeted therapy has brought survival benefit to NSCLC patients. So far there is no effective treatment for PMEC patients who cannot undergo surgery. Few studies had paid attention to driven genes such as EGFR and ALK. It was noted that the mutational frequency of EGFR in PMEC was $40 \%$ and mutations were all L858R mutation. A case of ALK-rearranged PMEC was reported by Wong et.al in 2009 [22-24]. In our retrospective cases, we identified ALK rearrangement in a non-smoking woman with high grade PMEC. However, due to the small scale of such report, no predictive markers have been found. More attention should be paid to investigate mutational profile of PMEC to provide alternative therapy for advanced patients, especillay MAML2 rearrangement.

\section{Conclusions}

Our study has provided supplementary clues for the identification of PMEC and updated the understanding of PMEC. Early diagnosis and complete surgical resection may promise a good prognosis. Combined TTF-1 and MAML2 will benefit the identification of pulmonary mucoepidermoid carcinoma from other lung tumors. Mutational profile of PMEC deserves further study. Considering that there is no effective treatment for cases accompanied with distant metastasis, driven genes of existing drugs such as EGFR and ALK were recommended to detect in clinic. However, as a retrospective study, it had inherent limitations due to the small size of sample. Meanwhile, some clinical data and follow-up information were not available. Future prospective randomized controlled trials and larger, multi-centric series are needed to overcome the challenges related to the accurate diagnosis and prediction of PMEC.

\section{Abbreviations}

PMEC: Pulmonary mucoepidermoid carcinoma

WHO: World Health Organization

CTDComputed tomography

CA125: Carbohydrate antigen 125

NSE: Neuron-specific enolase

CYFRA21: Cytokeratin 19 fragments

CA153: Carbohydrate antigen 153

SCCA: Squamous cell carcinoma antigen

CEA: Carcinoembryonic antigen 
AFP: Alpha fetoprotein

H\&E: Hematoxylin and eosin

PSGT: primary salivary gland-type tumors

PACC: primary adenoid cystic carcinoma

EMC: epithelial-myoepithelial carcinoma

PA: pleomorphic adenocarcinoma

TTF-1: Thyroid transcription factor-1

NSCLC: Non small cell lung cancer

ALK: Anaplastic lymphoma kinase

EGFR: Epidermal growth factor receptor

\section{Declarations}

\section{Availability of data and materials}

The datasets used and/or analyzed during the current study are available from the corresponding author on reasonable request.

Acknowledgements: Notapplicable.

\section{Ethics declarations:}

Authors' contributions $\square Y i$ Xiang and Jialei Wang contributed the central idea. Jing Zhang and Xiaoyan Chen analyzed most of the data, and wrote the initial draft of the paper. Zijun Qian and Ping Yue contributed to refining the ideas, carrying out additional analyses and finalizing this paper.

Funding: This study was supported by National Natural Science Foundation of China (Grant number 81672271), Shanghai Jiao Tong University Project and Shanghai Key Discipline for Respiratory Disease (Grant number 2017ZZ02014). The funders have played an important role in the design of the study and collection of data.

Ethics approval and consent to participate: The study was conducted under the approval by the Institutional Review Board. Ethical approval was granted by the clinical Research Ethics Committee of Shanghai Ruijin Hospital, Jiaotong University and Shanghai Cancer center, Fudan university. Signed informed consent was obtained from all induced patients for the acquisition.

Patient consent for publication: All patients signed consent for publication. 
Competing interests: The authors declared that they have no conflict of interest.

\section{References}

1. Hsieh CC, Sun YH, Lin SW, Yeh YC, Chan ML. Surgical outcomes of pulmonary mucoepidermoid carcinoma: A review of 41 cases. PLoS One. 2017; 12: e0176918.

2. Yamamoto T, Nakajima T, Suzuki H, Tagawa T, Iwata T, Mizobuchi T, Yoshida S, Nakatani Y, Yoshino I. Surgical treatment of mucoepidermoid carcinoma of the lung: 20 years' experience. Asian Cardiovasc Thorac Ann. 2016; 24: 257-261.

3. Lee GD, Kang DK, Kim HR, Jang SJ, Kim YH, Kim DK, Park SI. Surgical outcomes of pulmonary mucoepidermoid carcinoma: a review of 23 cases. Thorac Cardiovasc Surg. 2014; 62: 140-146.

4. Jiang L, Li P, Xiao Z, Qiu H, Zhang X, Xiao Y, Zhang B. Prognostic factors of primary pulmonary mucoepidermoid carcinoma: a clinical and pathological analysis of 34 cases. Int $\mathrm{J}$ Clin Exp Pathol. 2014; 7: 6792-6799.

5. Wang M, Ouyang S, Sun P, Li D, Huang G. Pulmonary mucoepidermoid carcinoma in Chinese population: a clinicopathological and radiological analysis. Int J Clin Exp Pathol. 2015; 8: 3001-3007.

6. Chen H, Zhang J, Qiu XJ, Wang J, Pei YH, Wang YL. Interventional Bronchoscopic Therapy in Adult Patients with Tracheobronchial Mucoepidermoid Carcinoma. Chin Med J (Engl).2017 Oct 20;130(20):2453-2458.

7. Ishikawa Y, Alvarez-Fernandez E, Aubry MC, Dacic S, Nicholson AG. Mucoepidermoid carcinoma. In:Travis WD, Brambilla E, Burke AP, Marx A, Nicholson AG, editors. WHO classification of tumors of the lung, pleura, thymus and heart. Lyon: IARC Press; 2015. pp. 99-100.

8. Travis WD. The 2015 WHO classification of lung tumors. 2014 Nov;35 Suppl 2:188.

9. Kumar V, Soni P, Garg M, Goyal A, Meghal T, Kamholz S, Chandra AB. A Comparative Study of Primary Adenoid Cystic and Mucoepidermoid Carcinoma of Lung. Front Oncol.2018 May 15;8:153.

10. PhD JRMM, Aubry MC, Lewis JE, Wampfler JA, Ms BAW, Midthun DE, PhD PYM, Cassivi SD. Primary salivary gland-type lung cancer. Cancer. 2007; 110: 2253-2259.

11. Cheng DL, Hu YX, Hu PQ, Wen G, Liu K. Clinicopathological and multisection CT features of primarypulmonary mucoepidermoid carcinoma. Clin Radiol.2017 Jul;72(7):610.e1610.e7.

12. Huo Z, Wu H, Li J, Li S, Wu S, Liu Y, Luo Y, Cao J, Zeng X, Liang Z. Primary Pulmonary Mucoepidermoid Carcinoma: Histopathological and Moleculargenetic Studies of 26 Cases. PLoS One. 2015; 10: e0143169.

13. Yamamoto T, Nakajima T, Suzuki H, Tagawa T, Iwata T, Mizobuchi T, Yoshida S, Nakatani $Y$, Yoshino I. Surgical treatment of mucoepidermoid carcinoma of the lung: 20 years' experience. Asian Cardiovasc Thorac Ann.2016 Mar;24(3):257-61.

14. Yousem SA, Hochholzer L. Mucoepidermoid tumors of the lung. Cancer. 1987 Sep 15;60(6):1346-52. 
15. Komiya T, Perez RP, Yamamoto S, Neupane P. Primary lung mucoepidermoid carcinoma: analysis of prognostic factors using surveillance, epidemiology and end results program. Clin Respir J. 2017 Nov;11(6):847-853.

16. Qin BD, Jiao XD, Liu K, Wu Y, He X, Liu J, Zang YS. Clinical, pathological and treatment factors associated with the survival of patients with primary pulmonary salivary gland-type tumors. Lung Cancer. 2018 Dec;126:174-181.

17. Jee KJ, Persson M, Heikinheimo K, Passador-Santos F, Aro K, Knuutila S, Odell EW, Makitie A, Sundelin K, Stenman G, Leivo I. Genomic profiles and CRTC1-MAML2 fusion distinguish different subtypes of mucoepidermoid carcinoma. Mod Pathol. 2013; 26: 213-222.

18. Saade RE, Bell D, Garcia J, Roberts D, Weber R. Role of CRTC1/MAML2 Translocation in the Prognosis and Clinical Outcomes of Mucoepidermoid Carcinoma. JAMA Otolaryngol Head Neck Surg. 2016; 142: 234-240.

19. Zhu F, Wang W, Hou Y, Shi J, Liu Z, He D, Bai C, Li S, Jiang L. MAML2 rearrangement in primary pulmonary mucoepidermoid carcinoma and the correlation with FLT1 expression. PLoS One. 2014; 9: e94399.

20. Roden AC, Garcia JJ, Wehrs RN, Colby TV, Khoor A, Leslie KO, Chen L. Histopathologic, immunophenotypic and cytogenetic features of pulmonary mucoepidermoid carcinoma. Mod Pathol. 2014; 27: 1479-1488.

21. Kalhor N, Moran CA. Pulmonary mucoepidermoid carcinoma: diagnosis and treatment. Expert Rev Respir Med. 2018 Mar;12(3):249-255.

22. Han SW, Kim HP, Jeon YK, Oh DY, Lee SH, Kim DW, Im SA, Chung DH, Heo DS, Bang YJ, Kim TY. Mucoepidermoid carcinoma of lung: potential target of EGFR-directed treatment. Lung Cancer. 2008; 61: 30-34.

23. Yu Y, Song Z, Gao H, Zhu L, Lu S, Zhang J, Luo Q. EGFR L861Q mutation is a frequent feature of pulmonary mucoepidermoid carcinoma. J Cancer Res Clin Oncol. 2012; 138: 1421-1425.

24. Wong DW, Leung EL, So KK, Tam IY, Sihoe AD, Cheng LC, Ho KK, Au JS, Chung LP, Pik Wong M; University of Hong Kong Lung Cancer Study Group. The EML4-ALK fusion gene is involved in various histologic types of lung cancers from nonsmokers with wild-type EGFR and KRAS. 2009 Apr 23.15;115(8): 1723-33.

\section{Tables}

Table 1Clinical data of 20 patients with pulmonary mucoepidermoid carcinoma 


\begin{tabular}{|c|c|c|c|}
\hline Parameters & Percentage & Parameters & Percentage \\
\hline Age,yr $(n=20)$ & & Negative & $14(82 \%)$ \\
\hline Mean & 45 & Distant metastasis $(n=12)$ & \\
\hline Range & $18-67$ & Positive & $1(8 \%)$ \\
\hline Gender $(n=20)$ & & Negative & $11(92 \%)$ \\
\hline Female & $8(40 \%)$ & Tumor markers $(n=9)$ & \\
\hline Male & $12(60 \%)$ & Present & $7(78 \%)$ \\
\hline Smoking $(n=13)$ & & CA125 & 3 \\
\hline Never & $8(62 \%)$ & NSE & 2 \\
\hline Have smoked & $5(38 \%)$ & CYFRA21-1 & 2 \\
\hline Symptoms $(n=10)$ & & CA153 & 1 \\
\hline Present & $8(80 \%)$ & SCCA & 1 \\
\hline Fever & 1 & CEA & 1 \\
\hline Cough & 7 & $\mathrm{AFP}$ & 1 \\
\hline Hemoptysis & 1 & Absent & $2(22 \%)$ \\
\hline Bone pain & 1 & Operation method(n=19) & \\
\hline Absent & $2(20 \%)$ & Lobectomy & $12(63 \%)$ \\
\hline Chest CT findings $(n=12)$ & & Sleeve resection & $1(5 \%)$ \\
\hline Atelectasis or obstructive pneumonia & $5(42 \%)$ & Pneumonectomy & $2(11 \%)$ \\
\hline A solitary nodule & $7(58 \%)$ & BiLobectomy & $4(21 \%)$ \\
\hline Bronchoscopy $(n=8)$ & & Adjuvant therapy $(n=12)$ & \\
\hline Neoplasm in lumen & $7(88 \%)$ & Present & $3(25 \%)$ \\
\hline Normal & $1(12 \%)$ & Radiotherapy only & 0 \\
\hline Tumor Location $(n=20)$ & & Chemotherapy only & 2 \\
\hline Right Lung & $13(65 \%)$ & Both & 1 \\
\hline Right u pper lobe & 2 & Absent & $9(75 \%)$ \\
\hline Right middle lobe & 5 & Follow-up time.mo(n=11) & \\
\hline Right lower lobe & 6 & Mean & 52 \\
\hline Left lung & $7(35 \%)$ & Rang & $8-126$ \\
\hline Left upper lobe & 4 & Prognosis $(n=11)$ & \\
\hline Left lower lobe & 3 & 3-year survival rate & $72 \%$ \\
\hline Lymph node metastasis $(n=17)$ & & Disease free & $8(80 \%)$ \\
\hline Positive & $3(18 \%)$ & Death & $2(20 \%)$ \\
\hline
\end{tabular}

Table2. Histopathological information of patients with pulmonary mucoepidermoid carcinoma 


\begin{tabular}{|c|c|c|c|}
\hline Parameters & Percentage & Parameters & Percentage \\
\hline Tumor grade(n=20) & & Pleural involvement $(n=11)$ & \\
\hline Low grade & $17(85 \%)$ & Positive & $4(36 \%)$ \\
\hline High grade & $3(15 \%)$ & Negative & $7(64 \%)$ \\
\hline TNM stage(n=12) & & Bronchus invasion $(n=13)$ & \\
\hline I-IIA & $9(75 \%)$ & positive & $2(15 \%)$ \\
\hline IIB-V & $3(25 \%)$ & negative & $11(85 \%)$ \\
\hline Resection margin $(n=14)$ & & TTF-1(n=10) & \\
\hline Positive & $0(0 \%)$ & Positive & 0 \\
\hline Negative & $14(100 \%)$ & Negative & $10(100 \%)$ \\
\hline Perineural invasion(n=6) & & P63(n=9) & \\
\hline Positive & $0(0 \%)$ & Positive & $6(67 \%)$ \\
\hline Negative & $6(100 \%)$ & Negative & $3(33 \%)$ \\
\hline Intravascualr embolus ( $\mathrm{n}=6$ ) & & $\operatorname{ALK}(n=3)$ & \\
\hline Positive & $2(33 \%)$ & Positive & $1(33 \%)$ \\
\hline Negative & $4(67 \%)$ & Negative & $2(67 \%)$ \\
\hline
\end{tabular}

Table3. Comparsion between patients with high and low grade of pulmonary mucoepidermoid. 


\begin{tabular}{|c|c|c|c|}
\hline Parameters & High grade & Low grade & P-value \\
\hline $\operatorname{Age}(n=20)$ & & & 0.035 \\
\hline Mean, range & $61(56-67)$ & $42(18-64)$ & \\
\hline $\mathrm{Ki}-67(\mathrm{n}=11)$ & & & 0.0005 \\
\hline Mean, range & $32 \%(15 \%-50 \%)$ & $3.6 \%(3 \%-10 \%)$ & \\
\hline Tumor size.cm(n=12) & & & 0.026 \\
\hline Mean, range & $4.7(3-6)$ & $2.6(1-4)$ & \\
\hline Gender $(n=20)$ & & & 1.000 \\
\hline Female & 1 & 7 & \\
\hline male & 2 & 10 & \\
\hline TNM stage $(n=12)$ & & & 0.127 \\
\hline I-IIA & 1 & 7 & \\
\hline IIB-IV & 2 & 1 & \\
\hline Local invasion $(n=19)$ & & & 0.546 \\
\hline Positive & 2 & 6 & \\
\hline Negative & 1 & 10 & \\
\hline Metastasis(n=17) & & & 0.465 \\
\hline Positive & 1 & 2 & \\
\hline Negative & 2 & 12 & \\
\hline
\end{tabular}

Local invasion included perineural invasion, blood vessel invasion, pleural involvement and bronchus invasion; Metastasis included lymph node, bone, abdominal and brain.

\section{Figures}



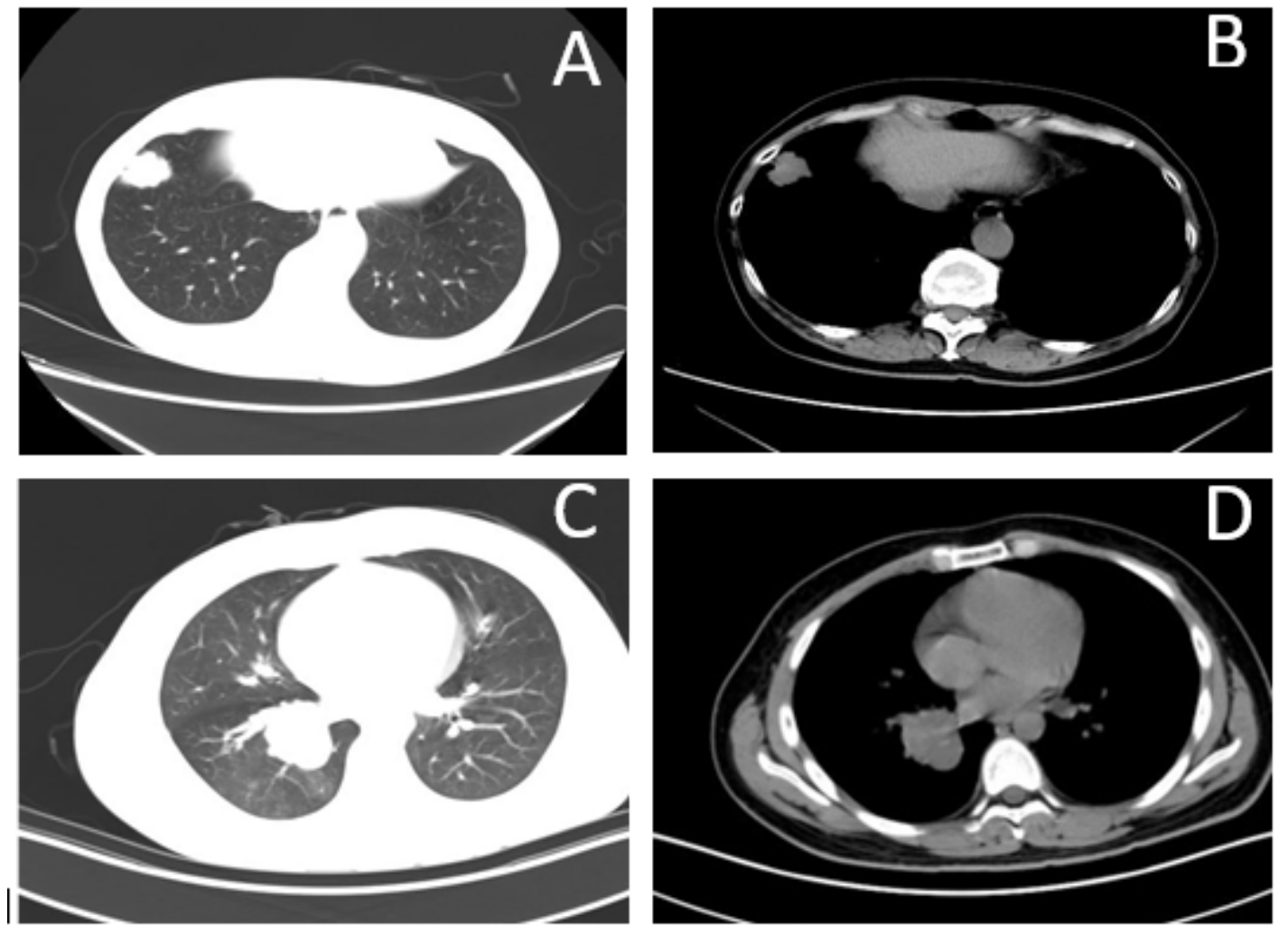

Figure 1

CT features of Pulmonary mucoepiermoid carcinoma. (A-B) Pulmonary mucoepiermoid carcinoma in a 64-year-old woman. CT shows a peripheral pulmonary nodule with slight lobulated and pleural involvement. (C-D) Pulmonary mucoepiermoid carcinoma in a 21-year-old man with a well-circumscribed mass at central site. 

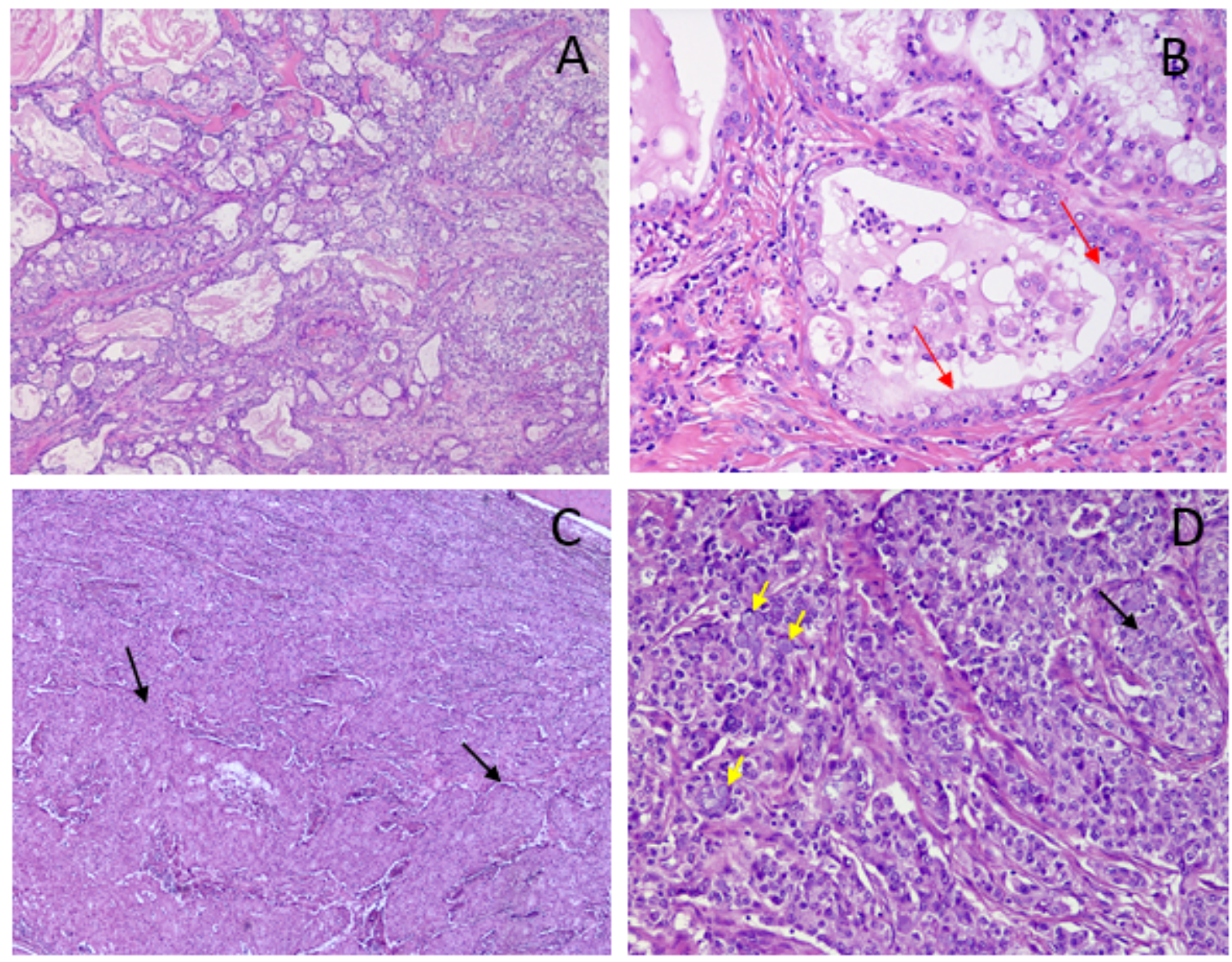

\section{Figure 2}

Hematoxylin-eosin staining images of low and high grade mucoepidermoid carcinoma. (A-B) Low-grade mucoepidermoid carcinoma showing glandular-like structures and mucin-secreting cells(red arrows);(C-D) High-grade mucoepidermoid carcinoma exhibiting evidently cellular atypia, islands of squamous cells(black arrows), with only scattered mucin 